\title{
The effect of light on appetite in healthy young individuals
}

\author{
M. AlBreiki, B. Middleton, J. Ebajemito and S. Hampton \\ Department of Biochemistry \& Physiology, School of Bioscience \& Medicine, Faculty of Health \& Medical Science, \\ University of Surrey, Guildford, GU2 7XH, UK
}

Appetite is controlled by a complex mechanism of hormones, neurotransmitters and neuropeptides both centrally or peripherally within the body. Few studies have demonstrated the effect of light in human subjects in modulating appetite. Light has been associated with a reduction in leptin ${ }^{(1)}$ and an increase in ghrelin ${ }^{(2)}$, both hormones associated with appetite. Light also influences melatonin which has been reported to be involved in appetite control in animals ${ }^{(3)}$. The aim of this study was to investigate the effects of light on appetite prior to and after a set meal in healthy subjects.

A favourable ethical opinion was obtained from the University Ethics Committee. Ten healthy participants ( 5 females and 5 males) mean age 22.4 years (SD 3.16) with body mass index (BMI) $22.73 \mathrm{~kg} / \mathrm{m}^{2}$ (SD 2.7) were randomised to a 2-way cross over design; dim light condition ( $<5$ lux) and bright light condition (>500 lux), separated by at least seven days. Each session started at 18:00 h and finished at 06:00 $\mathrm{h}$ the next day. Participants consumed an isocaloric and non-caffeinated evening meal (1066 Kcal, $38 \mathrm{~g}$ protein, $104 \mathrm{~g}$ $\mathrm{CHO}, 54 \mathrm{~g}$ fat, and $7 \mathrm{~g}$ fibre). The meal time was individualised based on melatonin onset (dim light melatonin onset (DLMO)) obtained from participants' 48 hour consecutive urinary collection. In order to assess subjective appetite, electronic visual analogue scales (VAS) were used (ProDiary). Appetite was assessed prior to and at regular intervals after the meal over a $5 \mathrm{~h}$ postprandial period, 3 major parameters were reported; hunger, fullness and prospective food consumption. Statistical analysis was carried out using Statsoft STATISTICA with two factor repeated measures ANOVA (time and treatment).

The preliminary results for perceived hunger and desire to eat are shown below. Statistical analysis showed significant differences in hunger and desire to eat between bright light and dim light treatment $(p=0 \cdot 014, p=0.016)$ respectively. There was no statistical significance in subjective fullness rating between the 2 conditions $(p=0 \cdot 68)$.
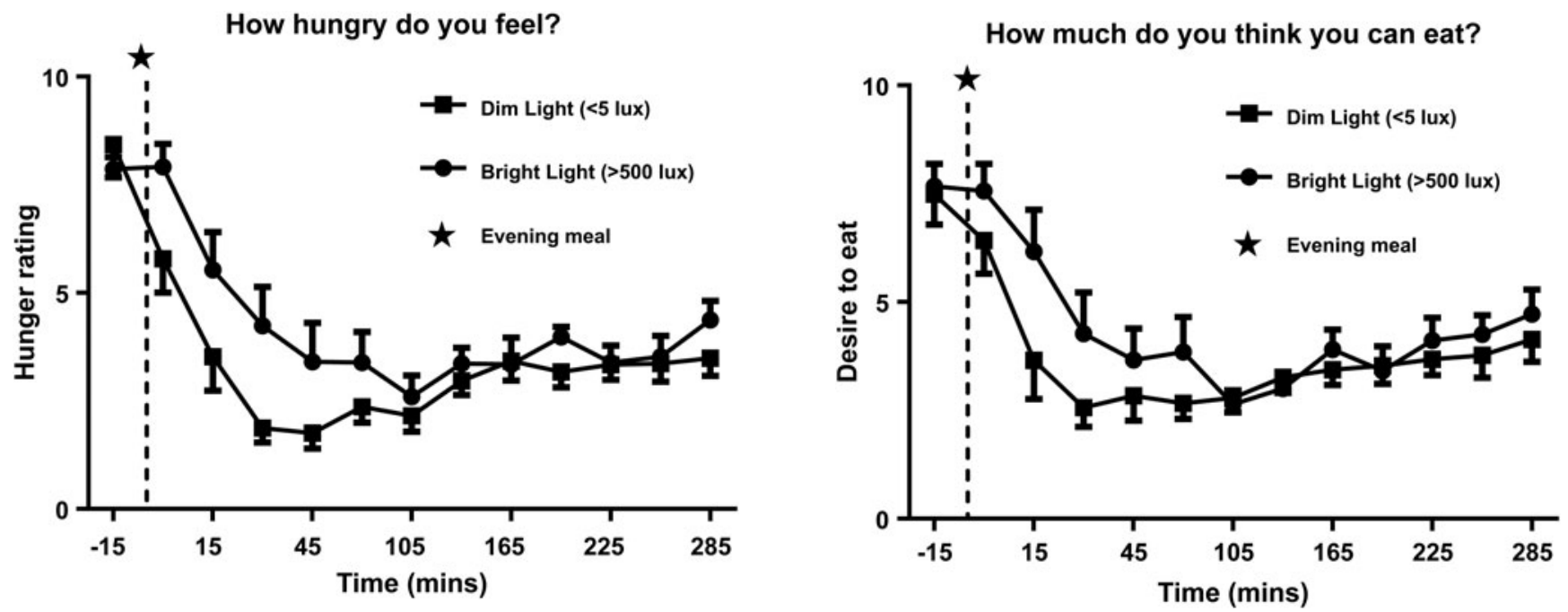

These preliminary results indicate that dim light decreases hunger and the desire to eat. The measurement of hormones involved in satiation such as leptin and ghrelin will provide further information on appetite control. These results support the idea that melatonin, which is present in dim light condition, may in part contribute to appetite control in humans. However, further investigations are required to confirm these findings.

1. Kalsbeek A, Fliers E, Romijin JA et al. (2001). Endocinology 142, 2677-2685.

2. Yildiz BO, Suchard MA,Wong ML et al. (2004) National Academy of Science 101, 10434-10439.

3. Piccinetti CC, Migliarini B, Olivotto I et al. (2010). Horm Behav 58, 780-785. 